\title{
Feature Scores Fusion for Chemical Class Recognition of Volatile Organic Compounds by Response Analysis of Surface Acoustic Wave Sensor Array
}

\author{
Sunil Kr. Jha \\ Department of Electronics \\ Graduate School of Information Science \& Electrical \\ Engineering, Kyushu University, 744 Motooka, \\ Fukuoka, 819-0395, JAPAN
}

\author{
Kenshi Hayashi \\ Department of Electronics \\ Graduate School of Information Science \& Electrical \\ Engineering, Kyushu University, 744 Motooka, \\ Fukuoka, 819-0395, JAPAN
}

\begin{abstract}
This paper presents fusion based pattern recognition approach of feature scores for chemical class identification of volatile organic compounds (VOCs) by response analysis of model surface acoustic wave (SAW) sensor array. Diverse features are generated by analysis of sensor array response using three feature extraction methods: principal component analysis (PCA), independent component analysis (ICA) and kernel principal component analysis (KPCA). Thereafter feature vectors are fused with three straightforward fusion strategies including i) summation, ii) multiplication and iii) combination of feature vectors. Chemical class recognition efficiency of fused feature is experimentally verified by feeding them to the input of support vector machine (SVM) classifier. Experimental outcomes are based on analysis of 12 data sets generated with SAW sensor model simulation, containing different intensity of noise and outliers. It has been observed that in research of three fusion schemes; fusion by summation of feature vectors achieves persistently highest correct chemical class recognition rate (average 90\%) of VOCs followed by combination and multiplication. Though in case of less noisy SAW sensor array response, fusion by combination of feature vectors results comparable class recognition efficiency to that of fusion by summation.
\end{abstract}

\section{General Terms}

Data Fusion, Pattern Recognition.

\section{Keywords}

Feature fusion, VOCs class recognition, SAW sensor, Electronic nose.

\section{INTRODUCTION}

A set of chemical sensors having varied selectivity in combination with a pattern recognition system is employed to design a machine that attempt to imitate mammalian smells capability popularly known as Electronic Nose (E-Nose) [1]. It is successfully used for the recognition of volatile organic compounds (VOCs) in various areas such as monitoring of food quality, environment, health and safety to security applications [1]. Polymer functionalized surface acoustic wave (SAW) oscillator is an impressive chemical sensor widely used in E-Nose operation [2]. Pattern recognition system of E-Nose extracts the hidden chemical identity information from sensor array response for qualitative and quantitative recognition of VOCs [3]. Major constraints of present E-Nose system are: the false alarm rate (assignment of VOCs to incorrect chemical class), low sensitivity, selectivity and reproducibility, high power consumption and response time etc. Most of the limitations can be reduced via the performance optimization of its sensing and pattern recognition system. Researchers are working on these two aspects of E-Nose, with objective to improve its chemical vapor sensing efficiency [4]. Amongst them, the development of an efficient and reliable pattern recognition software (i.e. information processing unit) for chemical vapor identification is the most limiting and costly aspects [1]. Pattern recognition system employs statistical, evolutionary and biologically inspired methods via three steps including: pre-processing, feature extraction and classification/quantification in sensor array signal processing [3].

As the complexity of the sensing environment increases information extracted by using a single technique at each steps of pattern recognition unit may not be sufficient and results in low recognition efficiency of E-Nose system. Data fusion approach is one of the solutions to overcome this limitation. It integrates the information extracted from different pattern recognition methods in order to achieve improved accuracy and more specific conclusion, than what could be achieved by using a single method [5-8]. The selection of architecture and procedure for data fusion strategy (i.e. at what stage and how the information should fuse) are data domain specific. It is a significant open research issue in present E-Nose system [7].

Three major architecture of data fusion is reported earlier in literature namely; data, or pre-processing level fusion, feature level fusion and decision, or classifier level fusion [7]. In data level fusion raw data set is preprocessed using different preprocessing method thereafter fused into a new single raw data set which is more informative than the original raw data set. In feature level fusion numerous feature extraction methods are used to obtain the diverse feature vector in different feature space, subsequently feature vectors are fused together to obtain common feature vectors. In decision level fusion data set is processed with independent classifiers after that a common decision is made by fusing the decision of each of the individual classifier. Mangai et al. in [9] have presented a brief summary of feature and decision level fusion schemes as implemented by researchers in different applications. Majority voting, weighted majority voting, behavior knowledge space, naïve Bayes, class set reduction and reordering, class conscious and indifferent are the main variety of decision level fusion methods reported earlier. Two methods are widely available in published literature for feature level fusion this includes the series and parallel combination. 
Initially data fusion methods have been developed and employed for the military applications including target and threat recognition, remote sensing, battlefield surveillance etc. $[10,11]$ Though at the present these methods have been widely used for civilian applications like in image processing, face recognition, speech processing, video classification and gene detection etc.[12-14] In E-Nose domain Dutta et al. [15] have used data level fusion for response analysis of tin oxide sensor array in freshness monitoring of eggs with 95\% accuracy. In another study Natale et al. have presented data level fusion of quartz resonators and metal oxide sensor array for quality evaluation of olive oil [16]. Li et al. [17] have reported feature and decision level fusion using Bayesian network in quality monitoring of apples by response analysis of E-Nose and z-Nose. In this study Principal component analysis (PCA) is used for feature extraction and probabilistic neural network (PNN) is used as classification method. Amongst the feature fusion schemes the dynamic selective fusion results best classification. A similar approach is presented by L. Rong et al. for wine classification [18]. In this study authors have employed fuzzy neural network for information fusion of E-Nose and E-tongue systems.

The intention of present study is to achieve the optimum performance of pattern recognition unit for E-Nose system intelligence by fusing the information from multiple feature extraction methods using simpler approaches. For this purpose we have implemented three feature fusion rules namely summation, multiplication and combination of feature vectors. These fusion schemes are not novel and presented by G. Marcialis and F. Roli in face recognition application [19]. However we are experimenting with these fusion rules first time for sensor array response analysis in gas sensing application. Also there are some fundamental variations between our approach and that presented in [19]. Authors in [19] have suggested the fusion schemes for PCA and linear discriminant analysis (LDA) distance vector (computed by using PCA and LDA feature vectors) but in present study we have employed these rules directly on PCA, Independent component analysis (ICA) and Kernel principal component analysis (KPCA) feature vectors. The objective of present study is to develop uncomplicated pattern recognition unit for E-Nose system. Comparison of direct and distance feature vectors and including feature vectors from some supervised extraction methods will be the subsequent part of this study.

Feature level fusion has specific benefits over the data and decision level fusion. It reduces the commensurate requirement of data level fusion also results more information gain compared to decision level fusion. Due to these advantages, it is widely acceptable in different applications. Therefore this study focuses on feature level fusion for chemical class recognition of VOCs by analyzing SAW model sensor array response. Fig. 1 shows the flowchart of suggested fusion schemes in detail. The feature vectors are generated with three unsupervised feature extraction methods including PCA, KPCA and ICA. These feature vectors are concatenated for fusion by three approaches: fusion by summation of feature vectors, multiplication of feature vectors and combination of feature vectors. Efficacy of fused feature vectors is validated by using support vector machine (SVM) classifier for class recognition of VOCs. Study is concluded by analyzing twelve sets of SAW sensor array response generated by using SAW sensor model simulation. Rest part of the paper is organized as follows. Detail description of data sets, feature extraction methods, feature fusion schemes and classification method are presented in section 2 and 3. Section 4 and 5 covers discussion on the experimental analysis outcomes on data sets. Final section presents the conclusion and future scope of study. .

\section{DATA SETS}

Data sets are generated theoretically by using the SAW sensor model response containing different intensity of additive noise and outliers. It is based on response of 11-element SAW sensor array (functionalized with different polymers) for 180 vapor samples belonging to six chemical classes. Each of the data sets contains response of chemical vapors from six chemical classes: trinitrotoluene (TNT), dinitrotoluene (DNT), dimethyl methylphosphonate (DMMP), water, toluene and benzene. Sensor array response is computed at 30 different concentration for each chemical vapors. Vapor concentration of chemicals varies in between ppth (parts per thousands) to ppt (parts per trillion). A summary of data sets are presented in Table 1. The basic difference amongst the data sets is due to value of noise and outliers incorporated in it. For instance the data set-IV contains additive Gaussian noise with mean value of $0 \mathrm{~Hz}$ and standard deviation $200 \mathrm{~Hz}$, whereas the data set-V includes Gaussian noise with random mean value in between $+50 \mathrm{~Hz}$ to $-50 \mathrm{~Hz}$ and standard deviation $50 \mathrm{~Hz}$. Detail description of experimental conditions, sensors and target VOCs can be seen in our earlier study [20].

Table 1. Summary of SAW sensor array data sets used in analysis.

\begin{tabular}{|c|c|c|c|c|}
\hline \multirow{2}{*}{$\begin{array}{l}\text { Data } \\
\text { sets }\end{array}$} & \multicolumn{2}{|c|}{ Noise level in Data sets } & \multicolumn{2}{|c|}{ Outlier level in Data sets } \\
\hline & $\begin{array}{l}\text { Mean Value } \\
\text { in } \mathrm{Hz}\end{array}$ & $\begin{array}{l}\text { Standard } \\
\text { deviation } \\
\text { in } \mathrm{Hz}\end{array}$ & Value in $\mathrm{Hz}$ & $\begin{array}{l}\text { Probability } \\
\text { of outlier } \\
\text { addition }\end{array}$ \\
\hline$I$ & 0 & 50 & Nil & Nil \\
\hline II & 0 & 100 & Nil & Nil \\
\hline III & 0 & 150 & Nil & Nil \\
\hline IV & 0 & 200 & Nil & Nil \\
\hline V & +50 to -50 & 50 & Nil & Nil \\
\hline$V I$ & Same as in $V$ & 100 & Nil & Nil \\
\hline VII & Same as in $V$ & 150 & Nil & Nil \\
\hline VIII & Same as in $V$ & 200 & Nil & Nil \\
\hline$I X$ & 0 & 10 & +50 to -50 & 0.75 \\
\hline$X$ & Same as in $I X$ & 10 & Same as in $I X$ & 0.80 \\
\hline$X I$ & Same as in $I X$ & 10 & Same as in $I X$ & 0.85 \\
\hline$X I I$ & Same as in $I X$ & 10 & Same as in $I X$ & 0.90 \\
\hline
\end{tabular}

\section{DATA PROCESSING METHODS}

A four steps pattern recognition system is used in present study for SAW sensor array response processing. It includes data preprocessing, feature extraction, feature fusion and classification. The analysis is done using functions available in packages of statistical computational language ' $R$ '. It is open access software. Short description of each of the steps is presented in following sub sections.

\subsection{Data Preprocessing}

Preprocessing of SAW sensor array response $\Delta f_{i j}$ (change in frequency of $j$-th sensor due to exposure of $i$-th vapor sample) involves three steps i) normalization with respect to frequency shift $\Delta f_{p}^{j}$ due to polymer coating followed by ii) logarithmic scaling as suggested in [21, 22] and finally iii) dimension autoscaling. After the normalization and logarithmic scaling the sensor array response is defined as $\Delta f_{i j} \leftarrow \log \left(\Delta f_{i j} / \Delta f_{p}^{j}\right)$ and employed for dimensional autoscaling. It is basically mean- 
centering followed by variance-normalization of sensor array response along sensors (dimensions) by treating vapors as random variable [23]

\subsection{Feature Extraction}

Three feature extraction methods namely: PCA, ICA and KPCA have been used for analysis of preprocessed SAW sensor array response. PCA is a linear and unsupervised feature extraction method. It transforms correlated sensor array response in measurement space into uncorrelated principal component (PC) space. The original sensor array response is projected along first few selected principal axes having highest eigenvalues. Projected sensor response vector is known as principal components or feature vector. This process reduces dimensionality of sensors response vector, facilitates in visualization of sensor response vector in two or three dimension and reduces further computation for chemical vapor identification. The detail description of method is given in [24-26]. 'stats' package [27] available in open source language ' $R$ ' is used for the PCA analysis.

KPCA is a nonlinear and unsupervised feature extraction method. It maps sensor array response from measurement space into high dimensional space (H-space) by using some nonlinear function termed as kernel function. Thereafter linear PCA is implemented on covariance matrix in $\mathrm{H}$-space for feature vector formation. Mathematical details of method can be seen in [24, 26]. 'kernlab' package [28] in $\mathrm{R}$ is used for the implementation of KPCA method.

ICA is a linear and unsupervised feature extraction method. It searches statistical independence by assuming non-Gaussian statistics of sensor array response. Method finds out orthogonal directions along which the projected sensors response has minimum correlation as well as the statistical dependency. The projection of sensor array response along these orthogonal directions is known as independent components and forms the feature vector. The detail mathematical description of method can be found in [29]. It is implemented by using the 'fast ICA' package [30] in ' $\mathrm{R}$ '.

\subsection{Feature Score Fusion}

This section describes the feature vector fusion schemes implemented in present study. Let $R=\left(R_{1}, R_{2}, R_{3}, \ldots \ldots . R_{n}\right)$ be the response vector of any chemical vapor from n-element SAW sensor array. Each of the response vectors in data set are projected into three feature spaces namely: PCA, KPCA and ICA.

Let $R^{P C A}=\left(R_{1}{ }^{P C 1}, R_{2}{ }^{P C 2}, R_{3}{ }^{P C 3}, \ldots \ldots . . R_{n}{ }^{P C n}\right)$

$R^{K P C A}=\left(R_{1}{ }^{K P C 1}, R_{2}{ }^{K P C 2}, R_{3}{ }^{K P C 3}, \ldots \ldots . . R_{n}{ }^{K P C n}\right)$

and $R^{I C A}=\left(R_{1}^{I C 1}, R_{2}{ }^{I C 2}, R_{3}{ }^{I C 3}, \ldots \ldots . . R_{n}{ }^{I C n}\right)$ be the corresponding feature vectors in PCA, KPCA and ICA spaces respectively.

For feature vector fusion three fusion rules have been implemented inspired from the study presented in $[19,31]$ as:

a. Feature vector summation

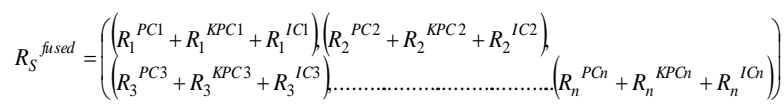

\section{b. Feature vector multiplication}

and KPCA we have selected only first three features. That is, its dimensionality is reduced from $11 \rightarrow 3$. Now each of the response vectors has only 3 dimensions in feature spaces. In

$$
R_{M}{ }^{\text {fused }}=\left(\begin{array}{l}
\left(\begin{array}{l}
\left.R_{1}{ }^{P C 1} \times R_{1}{ }^{K P C 1} \times R_{1}^{I C l}\right),\left(R_{2}{ }^{P C 2} \times R_{2}{ }^{K P C 2} \times R_{2}{ }^{I C 2}\right), \\
R_{3}{ }^{P C 3} \times R_{3}{ }^{K P C 3} \times R_{3}{ }^{I C 3}
\end{array}\right), \ldots \ldots \ldots \ldots \ldots \ldots \ldots \ldots \ldots . . \\
\left(R_{n}{ }^{P C n} \times R_{n}{ }^{K P C n} \times R_{n}{ }^{I C n}\right)
\end{array}\right)
$$

c. Feature vector combination

$$
R_{C}{ }^{\text {fused }}=\left(R_{1}^{P C 1}, R_{1}{ }^{K P C 1}, R_{1}^{I C 1}\right)
$$

First feature from each of the PCA, ICA and KPCA is selected for combination. Thus feature vectors generated by combination scheme have also three dimensions similar to the dimension of feature vector generated by summation and multiplication. In present study we have selected dimension of feature vectors both in single and fused feature space equal to 3 with objective to compare the performance of feature vectors in single and fused feature space and to reduce the dimensionality. It will also help in reducing the computation time of analysis as well as in comparative analysis amongst three fusion schemes.

\subsection{Support Vector Machine Classifier}

Support vector machine (SVM) method is introduced by Vapnik [32, 33] and summarized in reviews [34, 35]. In present study SVM is employed for class identification of chemical vapor by using the extracted feature vector in feature space. It is a supervised classification method. In binary class recognition problem, method builds an optimal separating hyperplane using the training data set. A class decision function is formulated on the basis of hyperplane. Chemical vapor is assigned to a particular chemical class based on sign of decision function. The hyperplane maximizes the interclass margin by using the quadratic programming (QP) optimization technique. The training data point close to hyperplane are used to measure the interclass margin and termed as support vector. In QP optimization kernel function is used to define the inner product of training data points. The multiclass SVM is an improved adaptation of binary class SVM. For multiclass identification using SVM, training data set is divided into combinations of several binary classes and the model is trained with each. In validation phase the test data points are classified with binary class trained SVM models. The final decision for class of an unknown data sample is made on the basis of majority voting of the binary class models. In the present study SVM is implemented as classifier by using the 'e1071' package [36] in 'R'.

\section{ANALYSIS RESULTS}

The goal of this study is to check the validity of proposed feature fusion schemes with the variety of SAW sensor array response data sets. For this purpose we have employed total 12 SAW sensor model generated data sets in analysis. Each of the data sets has dimension $(180 \times 11)$ and differs with others according to the level of noise and outliers incorporated. More details about the data sets and its analysis can be seen in Table 1, section 2 and Fig. 1. First of all each of the data sets is preprocessed using the methods discussed in section 3.1. After that feature vectors are generated using the three unsupervised feature extraction methods: PCA, ICA and KPCA with each of the data set independently. Novel feature vectors are generated by experimenting with three suggested fusion schemes.

Each of the data sets have 11-dimensional response vector in measurement space after feature extraction with PCA, ICA

feature fusion, only 3-dimensional feature vectors from each of the three feature spaces is used. This result three dimensional fused feature vectors by fusion schemes. 
Next to check the chemical class recognition ability of single as well as fused feature vectors SVM classifier is employed. Each of the data sets whether in single and fused feature space is divided first into training and test sets. $2 / 3^{\text {rd }}$ of data $(120$ samples) is used in training of SVM classifier and remaining $1 / 3^{\text {rd }}$ (60 samples) is used for its validation. Table 2 summarizes the SVM classification result of all the 12 data sets in both the single and fused feature spaces. A detail class wise confusion matrix for the classification of data set-I using the SVM classifier in single and fused feature space is presented in Table 3. Figs. 2-5 shows \% error in classification rate by SVM classifier for the data sets-I -XII in two kinds of feature spaces.

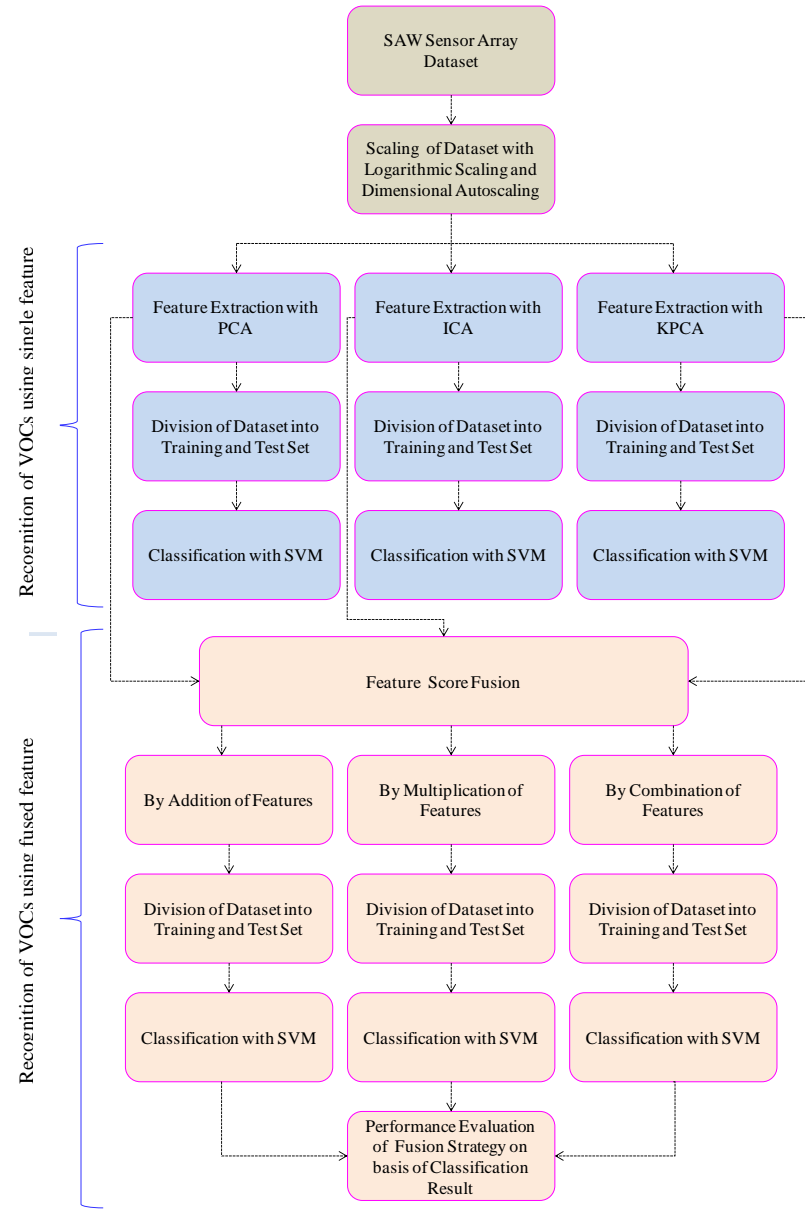

Fig 1: The computational analysis flow chart.

\section{DISCUSSION}

There are two main classes of fusion rules i) unsupervised rules (sum, product, minimum and maximum) and ii) supervised rules (SVM, bagging and boosting) [37]. Present study implements only unsupervised fusion rules since they can be easily executed without training. Another reason for selecting unsupervised fusion rules is that we have used 3 feature extraction methods, which transform the sensor array response in experimental measurement space to new mathematical feature space. Thus each of the feature set have different unit, hence a different set of weight is required for their performance comparison and simultaneous application in fusion schemes. Selection of optimal set of weights for each feature set is a complicated task. Unsupervised fusion rules (summation, multiplication and combination) used in present study resolve this issue by using equal weights to all the feature sets.
Table 2. Summary of SVM classification results for data sets-I-XII in single and fused feature spaces.

\begin{tabular}{|c|c|c|c|c|c|c|}
\hline \multirow[t]{3}{*}{$\begin{array}{l}\text { Data } \\
\text { Sets }\end{array}$} & \multicolumn{6}{|c|}{$\begin{array}{l}\text { Total number of samples correctly classified out of } 60 \text { in } \\
\text { validation phase by SVM classifier using the feature vectors in }\end{array}$} \\
\hline & \multicolumn{3}{|c|}{ Single Feature Space } & \multicolumn{3}{|c|}{ Fused Feature Space } \\
\hline & KPCA & ICA & PCA & $\begin{array}{l}\text { Multipli } \\
\text { cation }\end{array}$ & $\begin{array}{l}\text { Combi } \\
\text { nation }\end{array}$ & Addition \\
\hline$I$ & 35 & 47 & 55 & 32 & 55 & 56 \\
\hline II & 29 & 35 & 53 & 32 & 59 & 56 \\
\hline$I I I$ & 32 & 43 & 52 & 26 & 42 & 59 \\
\hline$I V$ & 28 & 40 & 49 & 27 & 54 & 55 \\
\hline$V$ & 32 & 38 & 49 & 21 & 45 & 52 \\
\hline$V I$ & 32 & 28 & 52 & 27 & 38 & 49 \\
\hline VII & 33 & 35 & 52 & 20 & 46 & 52 \\
\hline VIII & 20 & 33 & 31 & 22 & 40 & 47 \\
\hline$I X$ & 32 & 43 & 53 & 36 & 57 & 55 \\
\hline$X$ & 38 & 40 & 53 & 35 & 46 & 57 \\
\hline$X I$ & 32 & 13 & 26 & 23 & 38 & 37 \\
\hline$X I I$ & 29 & 15 & 34 & 23 & 34 & 43 \\
\hline
\end{tabular}

The summary of SVM classification results for all the 12 data set is presented in Table 2. Each digit in Table 2 represents the number of samples correctly identified out of 60 samples in validation phase of the SVM classifier using the feature vectors in single and fused feature space. The SVM classifier is trained independently with the each kind of the feature vectors in two spaces. After that it is validated with the feature vectors of each of the type to verify their chemical class recognition ability. It is evident from the Table 2 that the class recognition capability of feature vectors both in single and fused feature space decreases from data sets-I-XII. This is reasonable since as going from the data sets-I-XII the level of noise and outliers incorporated in data sets increases. It is also clear from the Table 2 that amongst the three kinds of feature vectors in single feature space, the performance of PCA feature vectors is better compare to ICA and KPCA feature vectors. The class recognition capability of KPCA feature vectors is worst. ICA feature vectors perform in between the PCA and KPCA feature vectors. As a consequence there is much scope in improving the class recognition capability of KPCA feature vectors if we want to use it as a robust feature extraction method in pattern recognition unit of E-Nose system. This will be the next target of our study that how to improve the class recognition capability of KPCA feature vectors by using feature fusion schemes. Amongst the three feature fusion schemes the feature vectors generated by addition performs best with correct classification rate more than the $90 \%$ except the data sets XI and XII. Since these two data sets have additional amount of noise and outlier as compare to rest of the data sets. The feature vectors generated by multiplication have poor class recognition capability with an average class recognition rate approximately $50 \%$. 
Table 3. Detailed SVM classification result for data set-I in single and fused feature spaces.

\begin{tabular}{|c|c|c|c|c|c|c|c|c|c|c|c|c|c|c|c|}
\hline \multicolumn{8}{|c|}{ Using KPCA feature vectors } & \multicolumn{8}{|c|}{ Using ICA feature vectors } \\
\hline \multirow{7}{*}{ 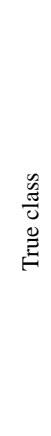 } & \multicolumn{6}{|c|}{ Predicted class } & \multirow{7}{*}{$\begin{array}{c}\text { Classification } \\
\text { Rate in }(\%) \\
\\
58.33\end{array}$} & \multirow{7}{*}{ 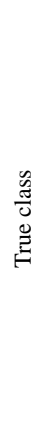 } & \multicolumn{6}{|c|}{ Predicted class } & $\begin{array}{c}\text { Classification } \\
\text { Rate in }(\%)\end{array}$ \\
\hline & 10 & 0 & 0 & 0 & 0 & 0 & & & 10 & 0 & 0 & 0 & 0 & 0 & \multirow{6}{*}{78.33} \\
\hline & 0 & 5 & 0 & 0 & 4 & 1 & & & 0 & 6 & 0 & 1 & 0 & 3 & \\
\hline & 0 & 0 & 10 & 0 & 0 & 0 & & & 0 & 0 & 10 & 0 & 0 & 0 & \\
\hline & 0 & 5 & 0 & 5 & 0 & 0 & & & 0 & 0 & 5 & 5 & 0 & 0 & \\
\hline & 0 & 7 & 0 & 0 & 3 & 0 & & & 0 & 4 & 0 & 0 & 6 & 0 & \\
\hline & 0 & 4 & 0 & 0 & 4 & 2 & & & 0 & 0 & 0 & 0 & 0 & 10 & \\
\hline \multicolumn{8}{|c|}{ Using PCA feature vectors } & \multicolumn{8}{|c|}{$\begin{array}{l}\text { Using fused feature vectors generated by multiplication of feature vectors in single } \\
\text { space }\end{array}$} \\
\hline \multirow{7}{*}{ 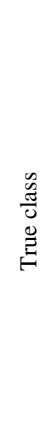 } & \multicolumn{6}{|c|}{ Predicted class } & $\begin{array}{c}\text { Classification } \\
\text { Rate in }(\%)\end{array}$ & \multirow{7}{*}{$\begin{array}{l}\tilde{z} \\
\frac{\tilde{z}}{0} \\
\tilde{D} \\
\tilde{E}\end{array}$} & \multicolumn{6}{|c|}{ Predicted class } & $\begin{array}{c}\text { Classification } \\
\text { Rate in }(\%)\end{array}$ \\
\hline & 10 & 0 & 0 & 0 & 0 & 0 & \multirow{6}{*}{91.67} & & 10 & 0 & 0 & 0 & 0 & 0 & \multirow{6}{*}{53.33} \\
\hline & 0 & 10 & 0 & 0 & 0 & 0 & & & 0 & 0 & 0 & 10 & 0 & 0 & \\
\hline & 0 & 0 & 10 & 0 & 0 & 0 & & & 5 & 0 & 4 & 0 & 0 & 1 & \\
\hline & 0 & 0 & 5 & 5 & 0 & 0 & & & 0 & 2 & 0 & 6 & 0 & 2 & \\
\hline & 0 & 0 & 0 & 0 & 10 & 0 & & & 0 & 1 & 0 & 4 & 5 & 0 & \\
\hline & 0 & 0 & 0 & 0 & 0 & 10 & & & 0 & 1 & 0 & 2 & 0 & 7 & \\
\hline \multicolumn{8}{|c|}{$\begin{array}{l}\text { Using fused feature vectors generated combination of by feature vectors in } \\
\text { single space }\end{array}$} & \multicolumn{8}{|c|}{ Using fused feature vectors generated by addition of feature vectors in single space } \\
\hline \multirow{7}{*}{$\begin{array}{l}\text { Oे } \\
\frac{\tilde{J}}{0} \\
\text { E } \\
E\end{array}$} & & & Predi & clas & & & $\begin{array}{l}\text { Classification } \\
\text { Rate in }(\%)\end{array}$ & \multirow{7}{*}{ 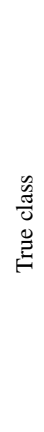 } & \multicolumn{6}{|c|}{ Predicted class } & $\begin{array}{l}\text { Classification } \\
\text { Rate in }(\%)\end{array}$ \\
\hline & 10 & 0 & 0 & 0 & 0 & 0 & \multirow{6}{*}{91.67} & & 10 & 0 & 0 & 0 & 0 & 0 & \multirow{6}{*}{93.33} \\
\hline & 0 & 10 & 0 & 0 & 0 & 0 & & & 0 & 10 & 0 & 0 & 0 & 0 & \\
\hline & 0 & 0 & 10 & 0 & 0 & 0 & & & 0 & 0 & 10 & 0 & 0 & 0 & \\
\hline & 0 & 0 & 0 & 10 & 0 & 0 & & & 0 & 0 & 4 & 6 & 0 & 0 & \\
\hline & 0 & 0 & 0 & 0 & 6 & 4 & & & 0 & 0 & 0 & 0 & 10 & 0 & \\
\hline & 0 & 0 & 0 & 0 & 1 & 9 & & & 0 & 0 & 0 & 0 & 0 & 10 & \\
\hline
\end{tabular}

Its performance looks equivalent to the KPCA feature vectors in single feature space. Thus the feature vectors generated by the multiplication appear an inappropriate fusion schemes in E-Nose applications. The feature vectors generated by the combination performs equally well as additive feature vectors in case of less noisy data sets (see the data sets-I-IV in Table 2) but as the noise level in data sets increases its performance deteriorate (see the data sets-V-XII in Table 2).

Finally it can be concluded from Table 2 that the PCA feature vectors have the best class identification capability as compare to ICA and KPCA feature vectors in single feature space and additive feature vectors generated by summation of feature vector in single feature space have best class identification capability in combination with SVM classifier.
Again if we compare the class identification capability of PCA in single feature space and additive feature vectors in fused feature space, additive feature vectors looks to have better recognition capability.

Table 3 contains detailed class wise identification information of chemical vapor for the data set-I by using three kinds of feature vectors in single feature space and another three kinds of feature vectors in fused feature space. $1^{\text {st }}$ column in each sub matrix of the Table 3 corresponds to TNT vapor class, $2^{\text {nd }}$ column corresponds to DNT vapor class, $3^{\text {rd }}$ column corresponds to DMMP vapor class, $4^{\text {th }}$ column corresponds to water vapor, $5^{\text {th }}$ column corresponds to toluene vapor class and $6^{\text {th }}$ column corresponds to benzene vapor class. The shells highlighted by colour in each of the sub tables denote the 
number of chemical vapor correctly assigned to their respective chemical class.

It is clear from the Table 3 that KPCA feature vectors results the lowest classification rate of $58.33 \%$, ICA feature vectors results classification rate of $78.33 \%$ and the PCA feature vectors results highest classification rate of $91.67 \%$ in single feature space. The confusion matrices for KPA, ICA and PCA shown in Table 3 indicate that by using KPCA feature vectors for classification there is confusion amongst the four chemical classes including DNT, water, toluene and benzene. While using the ICA features vectors for classification there is only confusion amongst three classes: DNT; water and benzene. The interclass confusion reduces to just one if PCA feature vectors are used for the class identification. That is if we use PCA feature vectors for the classification only water samples are misclassified. Thus amongst the feature vectors in single feature space, the PCA generated feature vectors result maximum correct classification rate and minimum class confusion when used in combination with SVM classifier.

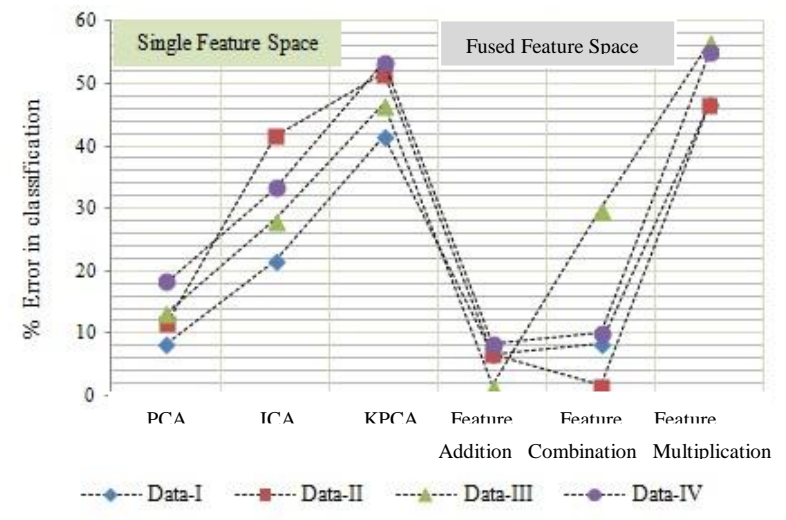

Fig 2: SVM classification error rate of data sets-I-IV in single and fused feature space.

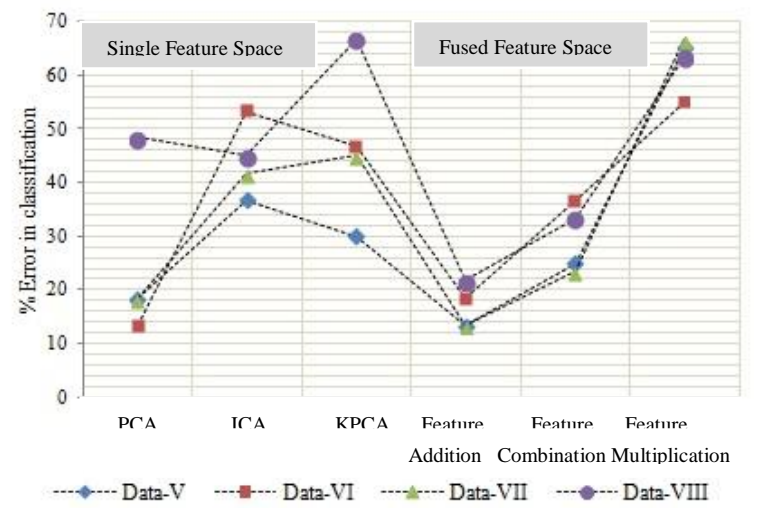

Fig 3: SVM classification error rate of data sets-V-VIII in single and fused feature space.

In fused feature space if feature vectors generated by multiplication is used for class recognition of chemical vapor it results only $53.33 \%$ of correct class identification rate and maximum confusion amongst the five classes: DNT; DMMP; water; benzene and toluene. Only the chemical vapor from the TNT class is correctly identified. Feature vectors generated by the combination shows some better performance with class recognition rate $91.67 \%$ and class confusion in only two chemical classes: toluene and benzene. The third kind of feature vectors generated with addition results the highest class recognition rate $93.33 \%$ with minimum confusion (only one chemical class water). Since water is a common interfering vapor so if we ignore it, the correct class recognition rate approaches to $100 \%$. Thus amongst the three kind of feature vectors in fused space, feature vectors generated by addition perform best for chemical class recognition as compare to the feature vectors generated by combination and multiplication. Also the additive features vectors perform better than any kind of feature vectors in single space. This is due to the nature of noise included in each of the data set and its elimination by feature extraction methods. The noise added in data sets is additive Gaussian in nature. Its effect is suppressed by each of the feature extraction methods when dimension of feature vector is reduced from $11 \rightarrow 3$ by eliminating the dimension of lower variance. Since the additive Gaussian noise affects more the feature vector dimension having lower variance than the dimension of higher variance. The effect of noise is further reduced in the feature vectors generated by the summation (since the residual noise is just added) as compare to the feature vectors generated by the multiplication (the residual noise is multiplied). This is the reason for better performance of additive feature vectors compare to the multiplicative feature vectors. Also the feature vectors generated by combination have the higher noise level as compare to the additive feature vectors and lower noise level as compare to the multiplicative feature vectors (since in combination the residual noise is combined in each of the feature vector dimensions). Thus the feature vectors generated by the combination have better chemical class recognition efficiency than the feature vectors generated by the multiplication and comparable to the feature vectors generated by summation.

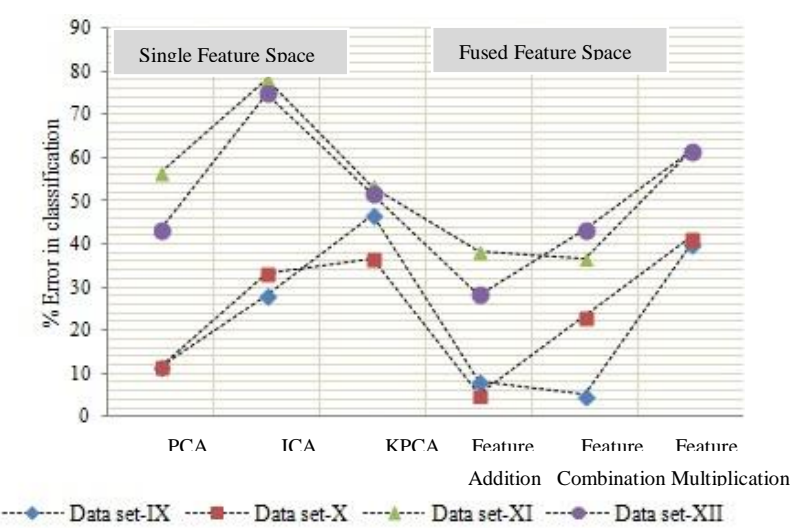

Fig 4: SVM classification error rate of data sets-IX-XII in single and fused feature space.

Figs. 2-4 present the error in class recognition rate of the SVM classifier in analysis of data sets-I-XII by using three kinds of feature vectors in single and three kinds of feature vectors in fused feature spaces. It is in favor of the results described in Table 2 and Table 3.

\section{CONCLUSION}

A simpler and effective feature fusion approach for chemical vapor class recognition is proposed based on response analysis of SAW sensor model in combination with SVM classifier. The present analysis shows that the chemical vapor recognition efficiency of SAW sensor array based E-Nose raises by using the fused feature vectors as compared to the feature vectors generated by single feature extraction method. 
Feature fusion by addition is found to be more effective fusion approach as compared with other two fusion approaches examined. As additive feature groups the discriminating information of chemical vapor from different feature spaces as well as eliminate the redundant information (noise) within the features. The objective of our next study is to improve chemical class recognition capability of KPCA feature vectors by using fusion strategies with objective to formulate this method more relevant in E-Nose signal processing.

\section{ACKNOWLEDGMENTS}

This research is funded by JSPS (Japan Society for the promotion of Science) related to the JSPS Grant-in-Aid for foreign researcher (24.02367). The author S.K.J gratefully acknowledges Director, A.K.G. Engineering College, Ghaziabad, India, Prof. P.K. Sharda for their consent and motivation to pursue this study and Anju S. Jha for her support.

\section{REFERENCES}

[1] Gardener, J.W. and Bartlett, P.N. 1999. Electronic Noses Principles and Application, New York, Oxford University Press.

[2] Arshak, K., Moore, E., Lyons, G.M., Harris, J. and Clifford, S. 2004. A review of gas sensors employed in electronic nose applications, Sensor Review 24, 181198

[3] Jurs, P.C., Bakken, G.A. and McClelland, H.E. 2000. Computational methods for the analysis of chemical sensor array data from volatile analytes, Chemical Review 100, 2649-2678.

[4] Rock, F., Barsan, N., and Weimar, U. 2008. Electronic nose: current status and future trends, Chemical Review $108,705-725$.

[5] Bloch, I. 1996. Information combination operators for data fusion: a comparative review with classification, IEEE Trans. Syst. Man Cyber. 26, 52-67.

[6] Varshney, P.K., 1997. Distributed Detection and Data Fusion, Springer-Verlag, New York.

[7] Hall, D.L. and Llinas, J. 1997. An introduction to multisensor data fusion, IEEE Proc. 85, 6-23.

[8] Goodman, I.R., Mahler, R.P.S. and Nguyen, H.T. 1997. Mathematics of Data Fusion, Kluwer Academic Publishers, Dordrecht, Netherland.

[9] Mangai, U.G., Samanta, S., Das, S. and Chaowdhury, P.R. 2010. A survey of decision fusion and feature fusion strategies for pattern classification, IETE Technical Review 27, 293-307.

[10] Smith, D. and Singh, S. 2006. Approaches to multisensor data fusion in target tracking: A survey, IEEE Trans. Know. Data Eng. 18, 1696-1710.

[11] Kleine-Ostmann, T. and Bell, A.E. 2001. A data fusion architecture for enhanced position estimation in wireless networks, IEEE Communications Letters 5, 343-345.

[12] Ben-Yacoub, S., Abdeljaoued, Y., and Mayoraz, E. 1999. Fusion of face and speech data for person identity verification IEEE Trans. Neural Networks 10 , 1065-1074.
[13] Jeon, B. and Landgrebe, D.A. 1999. Decision fusion approach for multi temporal classification IEEE Trans. Geoscience \& Remote Sensing 37, 1227-1233.

[14] Lanckriet, G.G., Bie, T.D., Cristianini, N., Jordan, M.I. and Noble, W.S. 2004. A statistical framework for genomic data fusion, Bioinformatics 20, 26262635 .

[15] Dutta, R., Hines, E.L., Gardner, J.W., Udrea, D.D and Boilot, P. 2003. Non-destructive egg freshness determination: an electronic nose based approach, Meas. Sci. Technol. 14, 190-198.

[16] Natale, C.D., Macagnano, A., Nardis, S., Paolesse, R., Falconi, C., Proietti, E., Siciliano, P., Rella, R., Taurino, A. and Amico, A.D'. 2001. Comparison and integration of arrays of quartz resonators and metal oxide semiconductors chemiresistors in the quality evaluation of olive oils, Sensors and Actuators B: Chemical 78, 303-309.

[17] Li, C., Heinemann, P., and Sherry, R. 2007. Neural network and Bayesian network fusion models to fuse electronic nose and surface acoustic wave sensor data for apple defect detection, Sensors and Actuators B: Chemical 125, 301-310.

[18] Rong, L., Ping, W. and Wenlei, H. 2000. A novel method for wine analysis based on sensor fusion technique, Sensors and Actuators B: Chemical 66 246-250

[19] Marcialis, G., and Roli, F. 2006. Decision level fusion of PCA and LDA based face recognition algorithms, International Journal of Image and Graphics 6, 239311.

[20] Jha, S.K. and Yadava, R.D.S. 2010. Development of surface acoustic wave electronic nose using pattern recognition system, Defence Science Journal 60, 364 376.

[21] Yadava, R.D.S. and Chaudhary, R. 2006. Solvation transduction and independent component analysis for pattern recognition in SAW electronic nose, Sensors Actuators B: Chemical 113, 1-21.

[22] Jha, S.K., Yadava, R.D.S. 2009. Preprocessing of SAW sensor array data and pattern recognition, IEEE Sensors Journal 9, 1202-1208.

[23] Osuna, R.G. and Nagle, H.T. 1999. Method for evaluating data-preprocessing techniques for odor classification with an array of gas sensor, IEEE Trans System Man Cybernetics: B 29, 626-632.

[24] Theodoridis, S. and Koutroumbas, K. 2006. Pattern Recognition $2^{\text {nd }}$ edition, Academic Press, San Diego, Chapter 4 and 6 .

[25] Smith, L.I. 2002. A tutorial on Principal Components Analysis, 1-26.

[26] Bishop, C.M. 2006. Pattern Recognition and Machine Learning, Springer, New York, USA.

[27] R Development Core Team, R: A language and environment for statistical computing. R Foundation for Statistical Computing, Vienna, Austria, ISBN 3900051- 07-0, 2008. http://www.R-project.org. 
[28] Karatzoglou, A., Smola, A., Hornik, K. and Zeileis, A. 2004. Kernlab - An S4 Package for Kernel Methods in R, Journal of Statistical Software 11, 120. http://www.jstatsoft.org/v11/i09.

[29] Hyvärinen, A., Karhunen, J. and Oja, E. 2001. Independent Component Analysis, John Wiley \& Sons, Canada.

[30] Marchini, J.L., Heaton, C. and Ripley, B.D. 2007. fastICA: FastICA Algorithms to perform ICA and Projection Pursuit. R package version 1.1-9.

[31] Fumera, G. and Roli, F. 2004. Analysis of error-reject trade-off in linearly combined multiple classifiers, Pattern Recognition, 1245-1265.

[32] Vapnik, V. 1979. Estimation of Dependences Based on Empirical Data [in Russian], Nauka, Moscow, [English translation], Springer Verlag, New York, 1982.
[33] Vapnik, V., 1995. The Nature of Statistical Learning Theory, Springer Verlag, New York.

[34] Christopher, J.C. and Burges, A. 1998. Tutorial on support vector machines for pattern recognition, Data Mining and Knowledge Discovery 2, 121-167.

[35] Gunn, S.R. 1998. Support Vector Machines for classification and regression, Technical Report, 1-66.

[36] Dimitriadou, E., Hornik, K., Leisch, F., Meyer, D. and Weingessel, A. 2008. e1071: Misc Functions of the Department of Statistics (e1071), TU Wien, R package version 1.5-18.

[37] Sadeghi, M.T., Samiei, M. and Kittler, J. 2010. Fusion of PCA-based and LDA-based similarity measures for face verification, EURASIP Journal on Advances in Signal Processing, 1-12. 